\title{
Choroidal Melanoma Simulating Adenoma of the Retinal Pigment Epithelium Arising at the Site of Congenital Hypertrophy of the Retinal Pigment Epithelium
}

\author{
Basil K. Williams Jr. ${ }^{a, b}$ Maura Di Nicola ${ }^{a, b} \quad$ J. Antonio Lucio-Alvarez ${ }^{a, c}$ \\ David R. Lally ${ }^{d}$ Carol L. Shields ${ }^{a}$ \\ a Ocular Oncology Service, Wills Eye Hospital, Thomas Jefferson University, Philadelphia, PA, USA; \\ ${ }^{b}$ Ocular Oncology Service, University of Cincinnati College of Medicine, Cincinnati, OH, USA; ' $A$ ssociación para \\ Evitar la Ceguera en México (APEC), Mexico City, Mexico; ${ }^{d}$ Retina Research Institute, New England Retina \\ Consultants, Springfield, MA, USA
}

\section{Established Facts}

- Adenoma and adenocarcinoma of the retinal pigment epithelium (RPE) can arise from congenital hypertrophy of the RPE and can simulate posterior uveal melanoma.

- Distinct clinical features of adenoma and adenocarcinoma of the RPE include abrupt elevation, intraretinal and subretinal exudation, and feeding and draining retinal vessels.

\section{Novel Insights}

- We describe a patient with an abruptly elevated choroidal melanoma arising at the site of congenital hypertrophy of the RPE and simulating RPE adenoma.

\section{Keywords}

Choroidal melanoma - Retinal pigment epithelium adenoma - Congenital hypertrophy of the retinal pigment epithelium · Malignant melanoma

\section{Abstract \\ Neoplasms of the retinal pigment epithelium (RPE) are rare tumors that can simulate choroidal melanoma, but clinical and imaging characteristics often differentiate these lesions.}

We report a 70-year-old male with an abruptly elevated pigmented lesion that arose at the site of congenital hypertrophy of the RPE and demonstrated associated exudation, as well as feeding and draining vessels, suggestive of RPE adenoma. Optical coherence tomography showed retinal elevation with serous retinal detachment adjacent to the mass, and ultrasonography revealed an abruptly elevated, moderately echodense mass of $6.4-\mathrm{mm}$ thickness. Fluorescein angiography showed early tumor hypofluorescence, late tumor hyperfluorescence with staining and leakage, and

\section{KARGER}

(c) 2019 S. Karger AG, Basel

E-Mail karger@karger.com

www.karger.com/oop
Carol L. Shields, MD

Ocular Oncology Service, Wills Eye Hospital

840 Walnut Street Suite 1440

Philadelphia, PA 19107 (USA)

E-Mail carolshields@gmail.com 
retinal vessels buried under the mass, suggestive of a retinal tumor. The patient was monitored with the presumed diagnosis of RPE adenoma, but 3 months later, the growth was documented and fine-needle aspiration biopsy revealed choroidal melanoma. Management with I-125 plaque radiotherapy was performed leading to tumor regression and a thickness of $4.6 \mathrm{~mm}$.

(c) 2019 S. Karger AG, Basel

\section{Introduction}

Benign and malignant neoplasms of the retinal pigment epithelium (RPE), adenoma and adenocarcinoma, respectively, are rare lesions that can simulate choroidal melanoma and historically have led to enucleation $[1,2]$. Both conditions are typically pigmented and appear as a solid mass in the posterior segment of the eye, but several additional features can clinically differentiate them, including abrupt elevation and related exudation with RPE tumors compared to dome or mushroom configuration and serous retinal detachment with melanoma. Melanoma is far more dangerous with risk for metastasis as compared to RPE tumors where metastatic disease is uncommon [3]. One important feature of RPE adenoma/adenocarcinoma is that this lesion can arise from an underlying congenital hypertrophy of the RPE (CHRPE). Herein, we describe a unique case of choroidal melanoma simulating an RPE neoplasm, arising directly at the site of CHRPE.

\section{Case Presentation}

A 70-year-old white male presented elsewhere with headaches and double vision. On examination, he was noted to have binocular horizontal diplopia that was worse in the left gaze and ptosis of the left upper eyelid. He was diagnosed with possible 6th nerve palsy and sent for computed tomography (CT) followed by magnetic resonance imaging (MRI) of the brain and orbits, which revealed a pituitary tumor expanding the sella turcica and extending into the suprasellar cistern with invasion of the left cavernous sinus. An incidental intraocular mass was noted in the nasal mid-periphery of the left eye (OS) (Fig. 1). The pituitary tumor was resected via a transphenoidal approach and proved to be an adenoma. The patient was then referred to our service for management of the intraocular mass.

On presentation, visual acuity measured $20 / 20$ in the right eye and 20/30 OS, and the intraocular pressure was within normal limits in both eyes. The right eye was unremarkable. Fundoscopy OS revealed an abruptly elevated, multilobulated pigmented mass with apical retinal invasion and measuring $8 \mathrm{~mm}$ in diameter with an underlying flat CHRPE with large lacunae (Fig. 2a). There was surrounding subretinal fluid, subretinal exudation at the posterior border of the lesion, and both a retinal artery and vein that appeared to be feeding and draining the lesion, respectively. B-scan
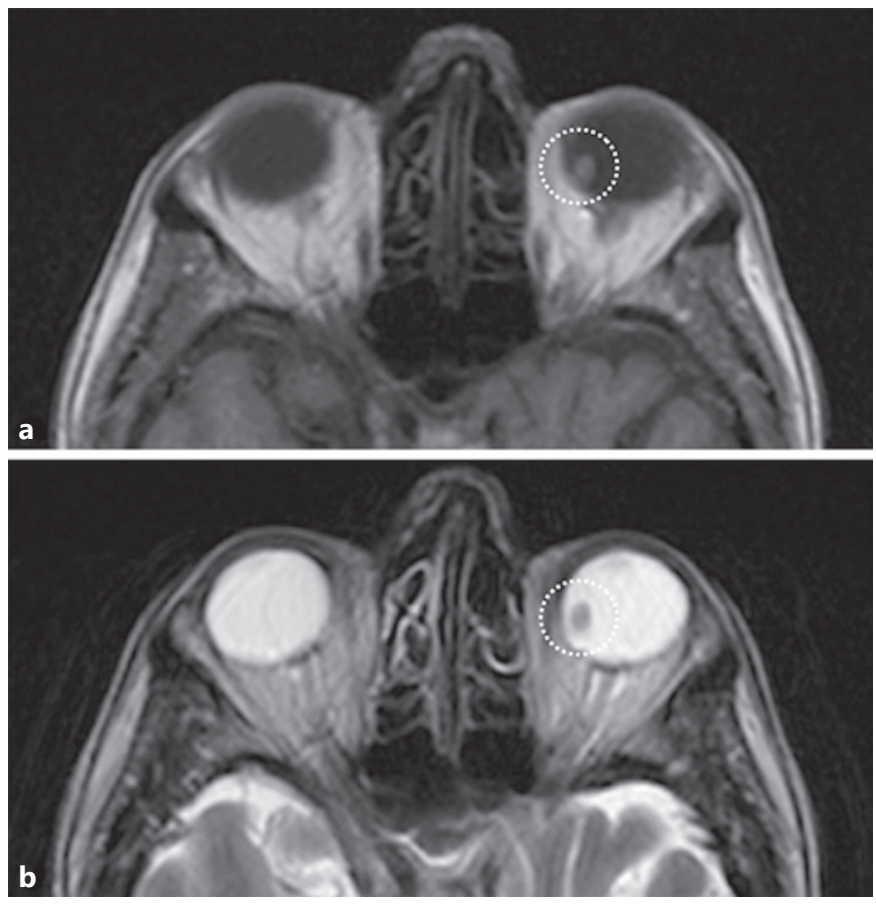

Fig. 1. MRI of the orbits demonstrating an intraocular mass in the nasal mid-periphery of the left eye (dotted white circle). The lesion appeared hyperintense in the T1-weighted image (a) and hypointense in the T2-weighted image (b). MRI, magnetic resonance imaging.

ultrasonography revealed an abruptly elevated, moderately echodense, and slightly mushroom-shaped lesion measuring $6.4 \mathrm{~mm}$ in thickness with localized subretinal fluid (Fig. 2c). Optical coherence tomography (OCT) of the macula showed an epiretinal membrane with lamellar hole OS (Fig. 2d). An OCT cut adjacent to the lesion demonstrated retinal elevation with subretinal fluid and hyperreflective spots, suggestive of retinal exudation (Fig. 2h). Fluorescein angiography (FA) revealed early hypofluorescence and late hyperfluorescence with leakage and staining (Fig. 2e, f).

Given the darkly pigmented mass with abrupt elevation, retinal invasion, and presence of underlying CHRPE, surrounding exudation, potential feeding and draining vessels, and a seemingly subretinal origin on OCT, the lesion was tentatively diagnosed as an RPE tumor, perhaps adenoma or adenocarcinoma. Observation was advised.

At the 3-month follow-up, the mass had grown appreciably in basal dimension (10-mm diameter) and thickness (7.2-mm thickness) (Fig. 3a, c), prompting fine-needle aspiration biopsy (FNAB) for both cytopathology and cytogenetics. Cytopathology revealed malignant melanoma cells, spindle B-cell type, supporting the diagnosis of choroidal melanoma. Cytogenetics revealed chromosome 1p loss, chromosome 3 loss, and chromosome 6 and 8 disomy, supporting the diagnosis of high-risk melanoma. Treatment with I-125 plaque radiotherapy to the tumor apex dose of $70 \mathrm{~Gy}$ over $103 \mathrm{~h}$ was provided. Screening for systemic metastasis was negative. On follow-up at 6 months, visual acuity was 20/60 OS with regression of the melanoma to $4.6 \mathrm{~mm}$ in thickness.
Williams Jr./Di Nicola/Lucio-Alvarez/ Lally/Shields 

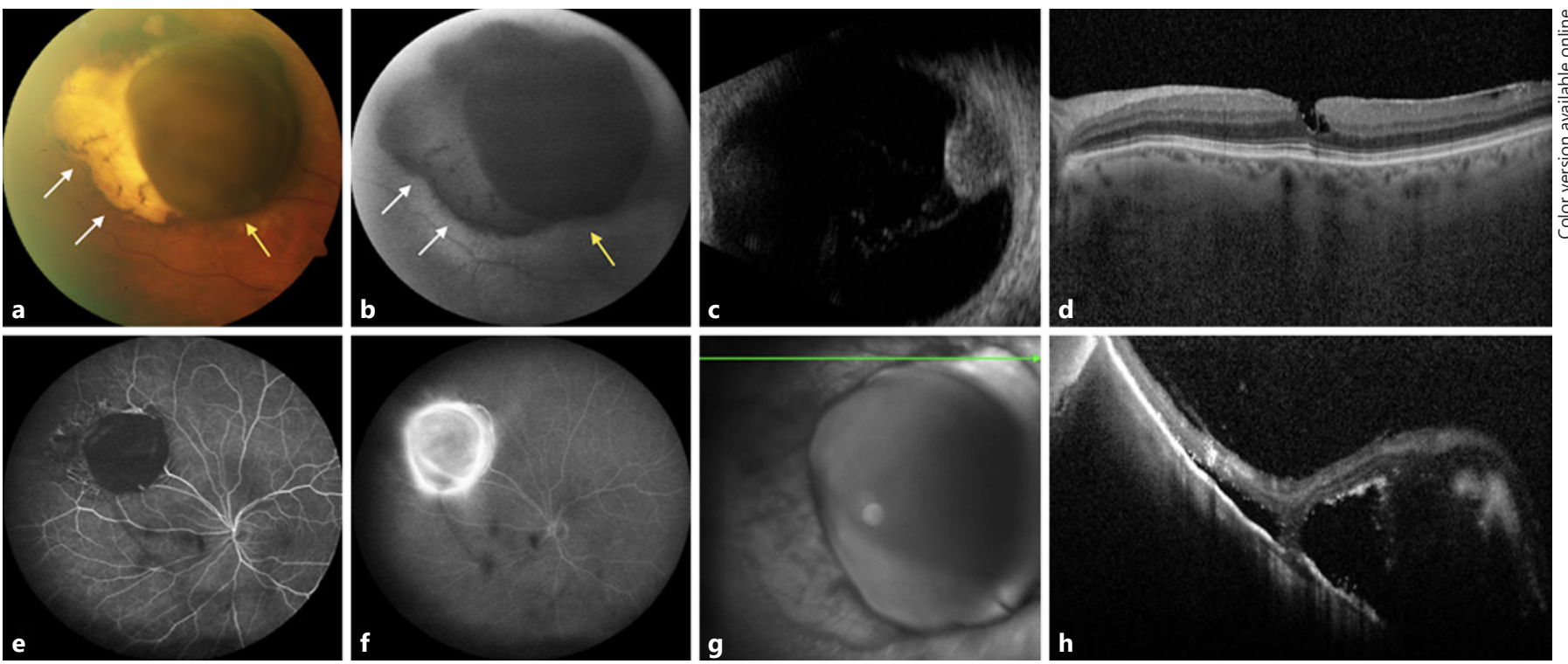

Fig. 2. Fundus photograph displaying an abruptly elevated, pigmented mass (yellow arrow) overlying an area of CHRPE with lacunae (white arrows) in the nasal mid-periphery of the left eye (a). Autofluorescence revealed the mass (yellow arrow), and underlying CHRPE (white arrows) appeared hypoautofluorescent (b). Bscan ultrasonography revealed an abruptly elevated, slightly mushroom-shaped mass measuring $6.4 \mathrm{~mm}$ in thickness with draped subretinal fluid and overlying vitreous debris, suggesting vitreous seeding or hemorrhage (c). Optical coherence tomography (OCT) demonstrated an epiretinal membrane and a lamellar hole (d). Fluorescein angiography depicted the lesion with early hypofluorescence (e) and late hyperfluorescence with leakage (f). Near-infrared image (g) with corresponding OCT cut (h) adjacent to the lesion showed retinal elevation with associated subretinal fluid and exudation. CHRPE, congenital hypertrophy of the retinal pigment epithelium; OCT, optical coherence tomography.
Fig. 3. At 3 months of observation, the mass (a) enlarged, measuring $7.2 \mathrm{~mm}$ in maximum thickness on ultrasonography (b), suggestive of choroidal melanoma. Wide-angle fundus photography at 6 months after I-125 plaque brachytherapy showed tumor regression with surrounding chorioretinal atrophy (c) and measuring $4.6 \mathrm{~mm}$ in thickness (d).
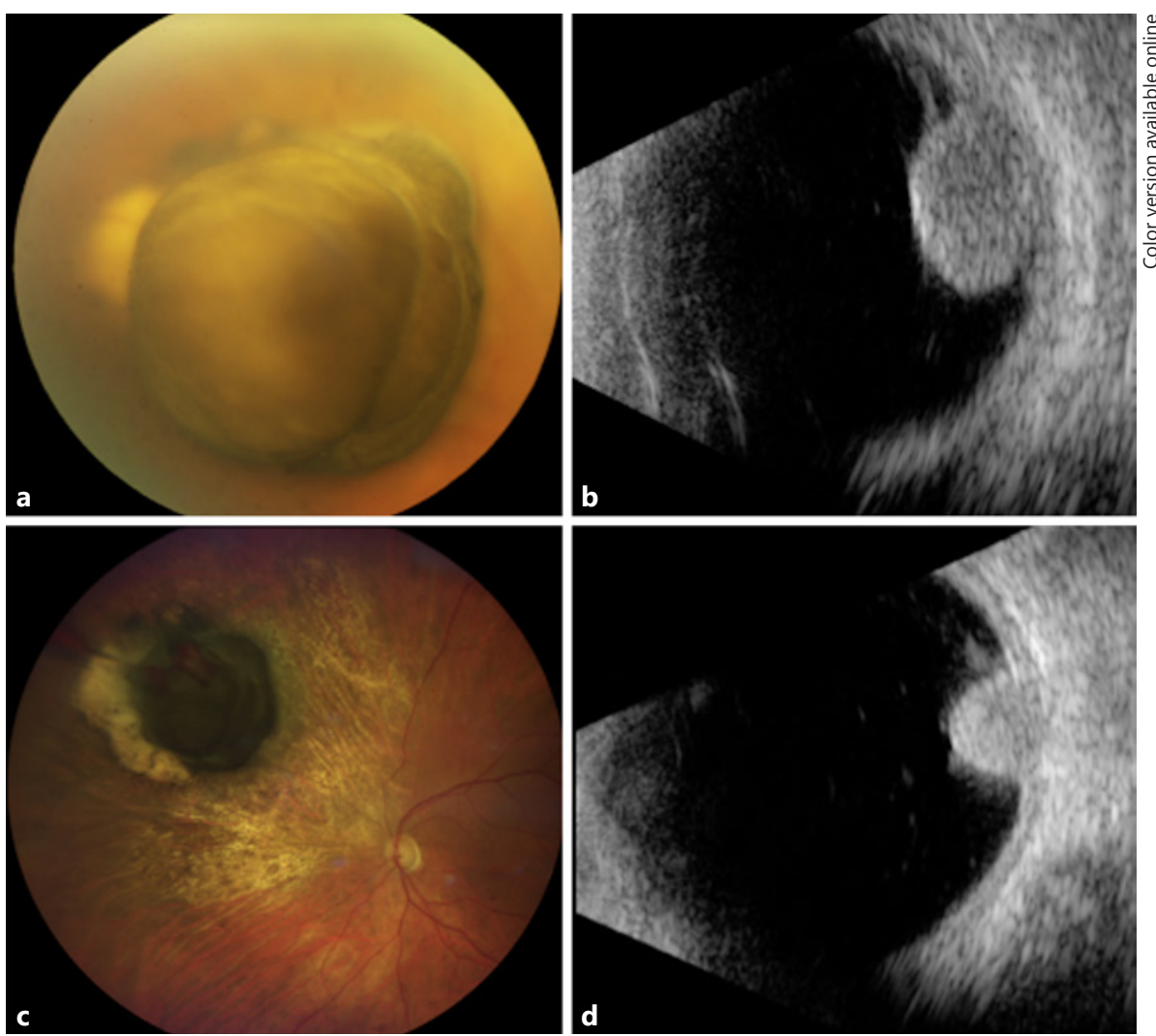

Choroidal Melanoma Simulating RPE Adenoma Arising from CHRPE
Ocul Oncol Pathol 2020;6:39-43 DOI: $10.1159 / 000501084$ 


\section{Discussion}

Adenoma and adenocarcinoma of the RPE are rare lesions that can simulate melanoma $[1,2]$. In a comprehensive series of 1,739 patients with lesions simulating choroidal melanoma, $13(<1 \%)$ represented RPE adenoma $[4,5]$. There are several features that differentiate RPE adenoma from choroidal melanoma. RPE neoplasms are often abruptly elevated as opposed to the dome or mushroom shape seen with melanoma. Intraretinal and subretinal exudation, along with remote macular epiretinal membrane, edema, or hole are a prominent feature with RPE tumors, but are rarely seen with melanoma [2]. As the overlying neurosensory retina is invaded, RPE neoplasms can acquire a retinal feeding artery and draining vein. Posterior uveal melanoma has been rarely noted to have a draining retinal vein, and retinal feeding artery has not been described $[6,7]$. Additionally, RPE neoplasms can arise from an underlying CHRPE, a finding not associated with melanoma $[2,8$, 9]. Of interest, when acquired RPE neoplasms arise from CHRPE, the majority have been at or anterior to the equator of the globe and associated with remote macular epiretinal membrane or cystoid macular edema, both of which were present in the current case.

There are further differences in these two tumors from an imaging standpoint. Using FA, RPE tumors often show early hypofluorescence and late hyperfluorescence, while uveal melanoma can demonstrate pinpoint leakage or prominent double circulation, especially in large mushroom-shaped lesions [2, 10]. Ultrasonography highlights the abrupt elevation, acoustic solidity and medium to high internal reflectivity of RPE neoplasms compared to the dome or mushroom appearance, acoustically hollowness, and low to medium internal reflectivity of uveal melanoma $[2,10]$.

The unique features of the choroidal melanoma in this case are that this tumor exhibited many clinical features more often associated with an RPE neoplasm, including arising at the site of CHRPE, abrupt elevation, surrounding exudation, and presence of feeding artery and draining vein, likely from retinal invasion. This tumor also demonstrated several imaging characteristics resembling RPE adenoma like early hypofluorescence from retinal invasion at the apex of the lesion and late hyperfluorescence with leakage and staining on FA, distant epiretinal membrane and lamellar hole on OCT, and a moderately echodense mass with abrupt elevation on ultrasonography. However, the rapid growth on follow-up suggested a choroidal melanoma over an RPE adenoma, leading to
FNAB for cytopathology and cytogenetics. Tissue diagnosis confirmed uveal melanoma and allowed for appropriate management with plaque brachytherapy. This case highlights the fact that despite the well identified clinical and imaging features of RPE neoplasms and posterior uveal melanoma, there may be considerable overlap in some lesions, making differentiation challenging. In these cases, FNAB may be of particular benefit to guide management and follow-up. Periodic observation for tumor growth in select cases is a reasonable approach as melanoma generally demonstrates growth at a much more rapid rate than RPE neoplasms.

In summary, posterior uveal melanomas can often be reliably differentiated from RPE neoplasms using clinical and imaging features. However, there are cases with overlapping findings, and the physician should have a low threshold to obtain a cytopathologic diagnosis via FNAB.

\section{Statement of Ethics}

The study complied with the guidelines for human studies and animal welfare regulations. The subject gave informed consent, and the study protocol was approved by the institute's committee on human research.

\section{Disclosure Statement}

The authors have no conflicts of interest to declare.

\section{Funding Sources}

Support provided by the Eye Tumor Research Foundation, Philadelphia, PA, USA (CLS). The funders had no role in the design and conduct of the study, in the collection, analysis, and interpretation of the data, or in the preparation, review, or approval of the manuscript.

\section{Author Contributions}

All authors were responsible for the conception and design of the work. B.K.W. and M.N. were responsible for drafting the work, and all authors were responsible for critical revision. The final version to be published was approved by all authors, who agree to be responsible for all aspects of the work.
Williams Jr./Di Nicola/Lucio-Alvarez/ Lally/Shields 


\section{References}

1 Ramahefasolo S, Soubrane G, Dhermy P, Godel V, Regenbogen L, Coscas G. Adenocarcinoma of retinal pigment epithelium. Br J Ophthalmol. 1987 Jul;71(7):516-20.

2 Shields JA, Shields CL, Gündüz K, Eagle RC Jr. Neoplasms of the retinal pigment epithelium: the 1998 Albert Ruedemann, Sr, memorial lecture, Part 2. Arch Ophthalmol. 1999 May;117(5):601-8.

3 Heindl LM, Naumann GO, Kruse FE, Holbach LM. Aggressive metastasising adenocarcinoma of the retinal pigment epithelium with trisomy 21. Br J Ophthalmol. 2008 Mar; 92(3):389-91.
4 Shields JA, Mashayekhi A, Ra S, Shields CL. Pseudomelanomas of the posterior uveal tract: the 2006 Taylor R. Smith Lecture. Retina. 2005 Sep;25(6):767-71.

5 Shields CL, Manalac J, Das C, Ferguson K, Shields JA. Choroidal melanoma: clinical features, classification, and top 10 pseudomelanomas. Curr Opin Ophthalmol. 2014 May; 25(3):177-85.

6 Johnson RN, Irvine AR, Char DH. Inferences from beading of a retinal vein draining a choroidal melanoma. Br J Ophthalmol. 1986 Oct; 70(10):764-5

7 Shields JA, Joffe L, Guibor P. Choroidal melanoma clinically simulating a retinal angioma. Am J Ophthalmol. 1978 Jan;85(1):67-71.
8 Trichopoulos N, Augsburger JJ, Schneider S. Adenocarcinoma arising from congenital hypertrophy of the retinal pigment epithelium Graefes Arch Clin Exp Ophthalmol. 2006 Jan; 244(1):125-8.

9 Shields JA, Eagle RC Jr, Shields CL, Brown GC, Lally SE. Malignant transformation of congenital hypertrophy of the retinal pigment epithelium. Ophthalmology. 2009 Nov: 116(11):2213-6.

10 Shields JA, Shields CL. Posterior Uveal Melanoma: Diagnostic Approaches. In: Shields JA, Shields CL, editors. Intraocular Tumors: An Atlas and Textbook. 3rd ed. Philadelphia: Wolters Kluwer; 2016. pp. 139-53. 